\title{
Modelo de Vida Útil para Estructuras Acero-Zinc Utilizadas en la Transmisión de Energía Eléctrica en Colombia
}

Fernando Villada, Juan D. Molina y Esteban Velilla

Universidad de Antioquia, Facultad de Ingeniería, Departamento Ingeniería Eléctrica,

Calle 67 No. 53-108, Oficina 19-441, Medellín-Colombia (e-mail: fvillada@udea.edu.co)

\begin{abstract}
Resumen
Se desarrolla un modelo de degradación por corrosión atmosférica para el acero y el zinc utilizados en el sistema de transmisión de energía eléctrica en Colombia. Se identifican tres aspectos importantes: la definición de la tasa de corrosión, la función distribución de probabilidad de la vida útil y las actividades (Inspección/mantenimiento) que favorezcan una mayor vida útil. El modelo identifica las funciones de pérdida de masa y grosor en el tiempo, lo que define límites de estados de degradación y falla los cuales son simulados mediante cadenas de Markov para evaluar la disponibilidad de la estructura y la frecuencia óptima de inspección y mantenimiento. Los resultados obtenidos permiten establecer zonas con menor vida útil y su impacto con la vida útil regulatoria. Se concluye que conocer los mecanismos de degradación permite de manera más acertada definir las frecuencias de inspección y mantenimientos respecto a la vida regulatoria.
\end{abstract} eléctrica

\section{Useful Life Model for Steel-Zinc Structures used in the Transmission of Electric Power in Colombia}

\begin{abstract}
An atmospheric corrosion degradation model for steel and zinc used in the transmission system of electric power in Colombia is developed. Three important aspects are identified: the definition of corrosion rate, the probability distribution function of useful life and the inspection/maintenance plan that favours a longer useful life. The model identifies mass loss and thickness loss functions, defining limits for degradation and fault modes which are simulated using Markov chains to evaluate the structure availability and the optimal inspection and maintenance frequency. The results allow establishing zones with lower service life and their impact on the regulatory life. It is concluded that knowledge of degradation mechanisms allows a better definition of inspection and maintenance frequencies under a regulatory life.
\end{abstract}

Keywords: steel-zinc structures, atmospheric corrosion, transmission lines, electric power 


\section{INTRODUCCIÓN}

El primer fenómeno que ocurre en los materiales para pasar a su estado natural es la corrosión. La corrosión atmosférica es la degradación y destrucción de los materiales metálicos, cuando estos interactúan con la atmósfera, es un proceso lento y ocurre de manera espontánea, el cual se puede prevenir o controlar, pero no se puede evitar pudiendo generar fallas en el sistema impactando la seguridad y la disponibilidad de los activos. Las empresas de transmisión de energía eléctrica cuentan con infraestructuras (Activos/Unidades Constructivas), las cuales están conformadas de manera general por líneas de transmisión -LT- y subestaciones -SE-.

En Colombia existe alrededor de $11,500 \mathrm{~km}$ de LT a 220 y $230 \mathrm{kV}$, y 2,410 km a $500 \mathrm{kV}$ (UPME, 2008), lo que supone grandes cantidades de material metálico, expuestos al efecto agresivo de las atmósfera o medios circundantes. Típicamente los activos de transmisión describen un modo de falla que se puede dividir en dos zonas, la primera con una tasa de falla constante y la segunda una zona de degradación en la cual la tasa de falla es creciente (Jiang et al., 2006). Algunos estudios (Endrenyi y Anders, 2006) destacan que del 100\% de fallas de un sistema de potencia, un $55 \%$ de fallas se deben a condiciones atmosféricas y un $14 \%$ a envejecimiento, siendo un problema importante sobre la infraestructura considerando que en Colombia existen estructuras con más de 20 años de instalación.

Entre los estudios de corrosión para el sector eléctrico se destacan autores como Mango (2001), quien analiza dos alternativas de protección para los radiadores de los transformadores, se compara si pintar (incluyendo mantenimiento) con el galvanizado de los radiadores. Concluyen que es mejor técnica y económicamente realizar el galvanizado para obtener una vida útil promedio de 32 años. Recientemente Linares et al. (2006), realizaron un análisis de falla por corrosión en conductores de aleación de aluminio 1350 (ACAR 1100) y 6201 (AACSR 1191) perteneciente a una línea de transmisión de $400 \mathrm{kV}$ ubicada en una zona costera e industrial, encontrando que la picadura es la falla que más se presenta siendo de gran impacto en la seguridad funcional del conductor. Adicionalmente Vera et al. (2006), evaluaron el efecto de los contaminantes atmosféricos en los conductores de energía eléctrica (AA6201) identificando que la picadura es el fenómeno que más ataca a los conductores de aluminio y el ambiente marino influye en la agresividad de la corrosión. En el caso de materiales como el cobre Vera et al. (2007), concluyen que la agresividad de la corrosión sobre este material no es significativa, lo que conlleva a una tasa de corrosión muy baja y para el acero galvanizado. Vera y Cañas (2005), establecen una mayor vida útil para aquellas estructuras que utilicen protección adicional como la pintura.

En Colombia se han adelantado proyectos (Minotas et al., 1996), buscando principalmente la identificación de grados de corrosión atmosférica. En CIC (2004), se establece que en términos generales, el mantenimiento en las estructuras es indispensable para preservar la vida útil de estas y esto es aún más crítico en el caso de infraestructura instalada en zonas con atmósferas marinas. Define que la vida útil de torres de transmisión galvanizada sometidas al ambiente marino, es del orden de 10 años, si no se realiza ningún mantenimiento a la misma y que una de las opciones para aumentar el tiempo de vida útil es pintar la estructura al menos 3 años después de instalada, acción que podría alargar el tiempo de vida útil hasta 35 años. En Maya (2007), se describen tres tipos de grados de corrosión y se define que la expectativa de vida de un elemento atacado por la corrosión depende del tiempo de servicio, la condición actual y la velocidad de corrosión. Adicionalmente, se propone una metodología para el manejo integral de la corrosión en LT el cual identifica 11 puntos de control de corrosión y establece un rango (6 - 12 años) de vida útil de los componentes de una LT de zonas altamente agresivas. En la Resolución 74 de 2005 (ww.creg.gov.co) se establece una franja de $30 \mathrm{~km}$ desde el litoral para reconocer por contaminación salina en el Nivel I. Se concluye que el ancho de la franja podría determinarse más exactamente si se lograra levantar un modelo predictivo (por ejemplo del tipo exponencial inverso) pero de cualquier forma, la distancia máxima para ser influenciados por el fenómeno salino no superaría los $30 \mathrm{~km}$ (Cabe destacar que a nivel del Sistema de Transmisión Nacional se reconoce una franja de hasta 100 km).

Dada la importancia de establecer mecanismos que aminoren el impacto por degradación en los activos, tanto en la confiabilidad, como en la disponibilidad de las mismas, este trabajo presenta el 
impacto de la corrosión atmosférica de manera que permita identificar diferentes alternativas de mantenimiento, reposición y planes de inversión futura en el contexto de la vida útil regulatoria.

\section{MATERIALES Y CORROSIÓN ATMOSFÉRICA}

Los factores más importantes a tener en cuenta de acuerdo a la atmósfera y sus efectos en el material son la temperatura, la humedad relativa, las precipitaciones anuales y el Tiempo de humectación -TdH-. Este último ha tomado mayor relevancia, debido a que indica el tiempo durante el cual la superficie del metal ha estado cubierta por una capa de electrolito, generalmente agua, que contiene contaminantes de la atmósfera y esto podría incrementar la tasa de corrosión. Sin embargo, la lluvia sirve para diluir contaminantes corrosivos y lavar la superficie del metal, conllevando a la disminución de la tasa de corrosión. La diferencia que se presenta en los valores de temperatura y humedad relativa de los diferentes ambientes influye en el comportamiento de la corrosión, debido a que el TdH ocurre a diferentes rangos de temperatura y por ende pueden ocurrir diferentes tasas de corrosión. A su vez la temperatura del metal y los valores de TdH pueden ser influenciados por la dirección del viento, debido a que cambian el contenido de contaminantes en la superficie del metal o por el contrario aumentan el nivel de deposición, generando una mayor erosión del metal.

La química de la atmósfera está estrechamente relacionada con la agresividad y los principales contaminantes son los cloruros $\left(\mathrm{Cl}^{-}\right)$y el dióxido de azufre $\left(\mathrm{SO}_{2}\right)$, estos pueden acelerar en gran magnitud la tasa de corrosión atmosférica, debido a que estas partículas suspendidas absorben agua lo que incrementa el periodo de $\mathrm{TdH}$ y por tanto el proceso corrosivo. La principal fuente de cloruros son partículas suspendidas de sal en entornos marinos, cloruro hidrogenado y hipocloruro de sodio en el entorno de plantas industriales. Los iones de cloruro $\left(\mathrm{Cl}^{-}\right)$son la principal fuente para acelerar la corrosión, y en particular ataque por picadura, debido a que penetran y destruyen la capa protectora de los metales.

Para el caso del sulfato, se encuentra en atmósferas urbanas e industriales, que en presencia de oxigeno se puede convertir en acido sulfúrico en la capa de humedad que se crea en la superficie de los metales, algunos metales tales como el aluminio y el zinc son relativamente resistentes a atmósferas con $\mathrm{pH}$ neutro, pero se corroen rápidamente en ambientes ácidos al igual que materiales inmersos en gases atmosféricos con alto número de iones. En el sector eléctrico los materiales comúnmente utilizados son el acero, el zinc, el cobre, el aluminio y aleaciones de estos, los materiales considerados en este trabajo son el acero y el zinc.

\section{MODELO DE VIDA ÚTIL}

Los modelos predictivos buscan determinar la curva de vida útil de las estructuras o su vida remanente, con el fin de cuantificar los impactos y efectos de los procesos de degradación que permitan identificar el impacto de los diferentes tipos de mantenimiento sobre la vida útil de la estructura considerando aspectos, tales como, la seguridad y la confiabilidad. Para identificar el modelo de degradación por corrosión atmosférica se identifican tres aspectos importantes, la definición de la tasa de corrosión, la función distribución de probabilidad para una determinada tasa de corrosión o vida útil y las actividades (mantenimientos) que incidan en la vida útil.

La variable fundamental para establecer la vida útil de una estructura es la tasa de corrosión (Kutz, 2005), de donde la vida útil de la estructura se puede estimar como la proporción entre la tasa de corrosión y el grosor mínimo de la estructura en el cual no se presenten fallas funcionales de la misma. La anterior definición se utiliza para tasas de corrosión uniforme y localizada. En la norma ISO 9223 (1992), se define la manera de encontrar la pérdida de masa por año de materiales estándar (acero y zinc). En general, la tasa de corrosión $T c\left(\mathrm{~g} / \mathrm{m}^{2}\right)$ de un metal después de un tiempo $t$ (años), depende directamente de la primera tasa anual de corrosión atmosférica $T c_{1},\left(\mathrm{~g} / \mathrm{m}^{2} \circ \mu \mathrm{m}\right)$ y es dependiente del tiempo (n), de acuerdo a la siguiente formula (Veleva y Kane, 2003):

$T c=T c_{1} t^{n}$ 
Donde $T c_{1}$ y $n$ dependen de los parámetros del metal y el clima. Los valores típicos de $n$ están en un rango de 0.5 a 1 , donde la mayoría de los valores son cercanos a la unidad. Para obtener una relación lineal entre $T c$ y $t$, se utiliza un modelo bilogarítmico, de la siguiente forma:

$\log T c=T c_{1}+n \log t$

El parámetro $T c_{1}$ depende de las propiedades del lugar, de ahí que se correlacione con las variables climáticas y la química de la atmósfera, teniendo en cuenta lo anterior, se correlaciona los niveles de contaminantes de dióxido de azufre y cloruros, mediante la siguiente expresión:

$T c_{1}=a_{1}+a_{2} T d H+a_{3} C_{S O 2}+a_{4} C_{C l}$

Donde $a_{1}, a_{2}, a_{3}, y a_{4}$ son coeficientes, y representan la tasa de deposición $\left(\mathrm{mg} / \mathrm{m}^{2} / \mathrm{día}\right)$ de dichos contaminantes.

Una parte importante de los sistemas a analizar, es definir su comportamiento, o su función de distribución que describa el proceso, en sistemas de deterioro algunos autores como Grall et al. (2002), utilizan la función Gamma; Gustavsen y Rolfseng (2005), utilizan la función Gauss, la distribución Gumbel's y la distribución normal y, Lefebvre (2003) la distribución Weibull.

Para el desarrollo del modelo se ha seleccionado la técnica de cadenas de Markov (IEC 61165, 2006). Autores como Anders et al. (2001); Tapias (2004); y Gustavsen y Rolfseng (2005) implementan cadenas de Markov sobre equipos de transmisión de energía. De manera similar Amari y McLaughlin (2004), desarrollan un modelo de mantenimiento basado en condición, el cual aplica procesos de decisión de Markov para identificar las actividades de mantenimiento a seguir y su programación. El modelo desarrollado en este trabajo consiste en identificar el mecanismo de degradación con el fin de establecer la vida útil de los materiales e identificar que actividades realizar sobre la infraestructura, ya sea de un material o un grupo de materiales, por ejemplo una torre de transmisión, y sus respectivas funciones de pérdida de masa y valoración del grosor en el tiempo, lo que permitiría definir límites de falla funcional o falla catastrófica. Dichos límites tienen asociada una incertidumbre, de ahí que para un determinado valor de grosor o vida útil (tiempo) se asume un comportamiento basado en una distribución de probabilidad. Para establecer la vida media útil perteneciente a cada zona se asume una distribución Weibull y se obtiene una función distribución de probabilidad mediante la simulación de aleatorios implementados en MATLAB $®$ R. Para cada zona se implementó su respectiva cadena de Markov en función de la vida útil obtenida de las funciones de degradación y la duración en cada estado para obtener la tasa de cambio y de esta manera poder evaluar la disponibilidad $A(t)$ del sistema. El modelo se implementó en Relex ${ }^{\circledR}$ Reliability Studio 2007 v2 -Evaluation-.

Para establecer la frecuencia óptima de inspección, mantenimiento correctivo y preventivo se implementó el modelo propuesto por Amari y McLaughlin (2004), el cual utiliza la técnica de Chapman-Kolmogorov (Amari y McLaughlin, 2004; Siqueira, 2004) para establecer una solución analítica a las cadenas de Markov. Se asume que el tiempo de inspección y de reemplazo es muy pequeño comparado con el tiempo total del sistema o componente. La tasa de inspección óptima (tiempo medio entre inspecciones) se halla mediante el método de bisección al establecer la tasa a la cual la disponibilidad es la mayor. Para el caso del tiempo medio entre mantenimientos correctivos y/o preventivos, se establece la probabilidad de que se encuentre en el estado de falla y la probabilidad de que se encuentre en el estado de mantenimiento. El tiempo medio entre inspecciones $\mathrm{MTBI}$, el tiempo medio entre mantenimientos correctivos MTBCM, el tiempo medio entre mantenimiento preventivo MTBPM se calcula según Amari y McLaughlin (2004). El modelo fue implementado en MATLAB ${ }^{\circledR} \mathrm{R}$.

En el marco del proyecto de investigación (COLCIENCIAS/CREG, 2008) se recolectaron variables atmosféricas del territorio nacional, tales como, temperatura, humedad relativa, altitud, y $\mathrm{TdH}$. Se identificaron 114 puntos, y empleando métodos estadísticos de análisis de cluster se agruparon en 6 familias de diferente agresividad. De cada una de estas familias se eligieron 2 sitios, para un total de 12 puntos representativos para realizar mediciones de corrosividad atmosférica durante 14 meses (COLCIENCIAS/CREG, 2008). 
Para establecer las funciones de degradación de cada uno de los materiales (acero y zinc) se reagruparon los puntos de medida en función de los datos de contaminantes obtenidos para un periodo de un año y se obtuvieron las siguientes zonas (Z): Z1 de Altos cloruros y sulfatos medios, Z2 de altos contenidos de sulfatos, Z3 de sulfatos moderados a bajo y la zona 4 de bajo contenido de cloruros y sulfatos. Partiendo de esta clasificación se procedió a identificar las funciones para cada una de las zonas y dentro de estas zonas para cada uno de los materiales (acero y zinc). La función de corrosión $\left(T_{C}\right)$ del acero para predicciones menores a 10 años se rige bajo la siguiente expresión:

$\mathrm{T}_{\text {CAcero }}=\frac{\mathrm{PMZ}_{\mathrm{n}}}{0,2358 \cdot \mathrm{t}}$

En la Tabla 1 se describen los factores de pérdida de masa ( $P M Z n$ ) para las cuatro zonas establecidas y las funciones de corrosión para predicciones iguales o mayores a 10 años (COLCIENCIAS/CREG, 2008). La función de corrosión $\left(T_{C}\right)$ del zinc para predicciones menores a 10 años se rige bajo la siguiente expresión:

$\mathrm{T}_{\text {CZinc }}=\frac{\mathrm{PMZ}_{\mathrm{n}}}{0,2142 \cdot \mathrm{t}}$

En la Tabla 2 se describen los factores de pérdida de masa (PMZn) para las cuatro zonas establecidas y las funciones de corrosión para predicciones iguales o mayores a 10 años. En el caso del zinc para las zonas 2 y 4, la función de corrosión para predicciones menores a 10 años es igual a la función de predicción igual o mayor a 10 años (COLCIENCIAS/CREG, 2008). Los parámetros y consideraciones utilizadas en las funciones se describen de la siguiente manera: $t$, es el tiempo en años; $\mathrm{P}$, es el tiempo en bimeses; $\mathrm{TdH}$, aumenta en la misma cantidad para todo el periodo de evaluación en 203,58 días. El área superficial en $\mathrm{m}^{2}$ es de 0,03 , la densidad $\left(\mathrm{g} / \mathrm{cm}^{3}\right)$ del acero y el zinc son de 7,86 y 7,14 , respectivamente.

Tabla 1: Función de corrosión y factores de pérdida de masa del acero.

\begin{tabular}{|c|c|c|}
\hline & PM para $\mathrm{t}<10$ años & $T_{C}$ para $\mathrm{t} \geq 10$ años \\
\hline $\mathrm{Z} 1$ & $\frac{26.1698}{1+12.6337 \cdot \mathrm{e}^{-0.804555 \cdot(0.9095 \cdot \mathrm{P}-0.6381)}}$ & $8.556 \cdot \mathrm{t}^{0.63}$ \\
\hline $\mathrm{Z} 2$ & $\frac{23.2509}{1+3.6444 \cdot \mathrm{e}^{-0.185294 \cdot(0.903 \cdot \mathrm{P}+0.3881)}}$ & $44.536 \cdot \mathrm{t}^{0.4}$ \\
\hline $\mathrm{Z} 3$ & $0.801176+0.433684 \cdot\left(0.9185 \cdot \mathrm{P}-0.6739^{1 / 2}\right)^{2}$ & $12.956 \cdot \mathrm{t}^{0.58}$ \\
\hline $\mathrm{Z} 4$ & $\frac{3.83184}{1+4.98507 \cdot \mathrm{e}^{-0.0117863 \cdot((\mathrm{TdH}+\mathrm{t}) / 2))}}$ & $13.623 \cdot \mathrm{t}^{0.3}$ \\
\hline
\end{tabular}

Tabla 2: Función de corrosión y factores de pérdida de masa del zinc.

\begin{tabular}{|c|c|c|}
\hline & PM para $t<10$ años & $T_{\text {c para } t \geq 10 \text { años }}$ \\
\hline Z1 & $\frac{0.774665}{1+5.39455 \cdot \mathrm{e}^{-0.853295 \cdot(0.9095 \cdot \mathrm{P}-0.6381)}}$ & $3.103 \cdot \mathrm{t}^{0.97}$ \\
\hline Z2 & $1.369 \cdot \mathrm{t}^{0.9}$ & $0.731 \cdot \mathrm{t}^{0.9}$ \\
\hline Z3 & $0.0153304+0.000469505 \cdot\left(0.9185 \cdot \mathrm{P}-0.6739^{2}\right)^{1 / 2}$ & \\
\hline Z4 & $1.099 \cdot \mathrm{t}^{0.8}$ & \\
\hline
\end{tabular}


Con base en el modelo descrito anteriormente, se identificará la vida útil de cada material dependiendo de la zona y su comportamiento para varias estrategias de recubrimiento, es decir, en el caso del acero galvanizado el comportamiento de la vida útil en función de la capa de zinc (para diferentes grosores).

\section{RESULTADOS Y DISCUSIÓN}

Los datos descritos anteriormente permiten caracterizar los 21 puntos de medida y el respectivo comportamiento de los materiales, de manera que podamos aproximar el comportamiento de los materiales en otras zonas que no hicieron parte de la experimentación. Con base en las funciones de corrosión se estableció la vida útil de los materiales para cada una de las zonas. Considerando que en las estructuras de líneas de transmisión se utilizan grosores de $3 / 16$ a $1 / 2$ pulgada (Molina, 2008) se utilizó el grosor mínimo 3/16 para establecer el mayor impacto sobre la vida útil en función del grosor y el tiempo. En la Fig. 1 y Fig. 2., se observa la pérdida de grosor en el tiempo del acero y el zinc, respectivamente. (Se asume que la pérdida de grosor se realiza de manera uniforme sobre las placas instaladas).

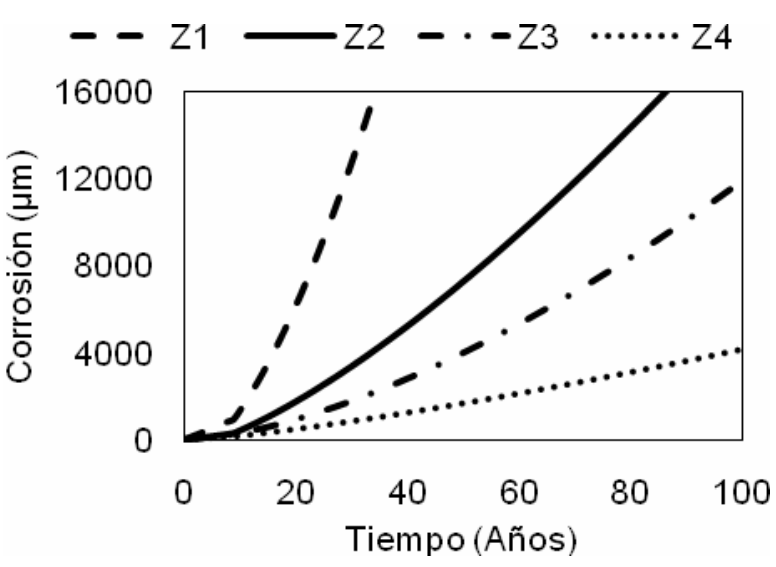

Fig. 1: Corrosión $(\mu \mathrm{m})$ del acero por zona.

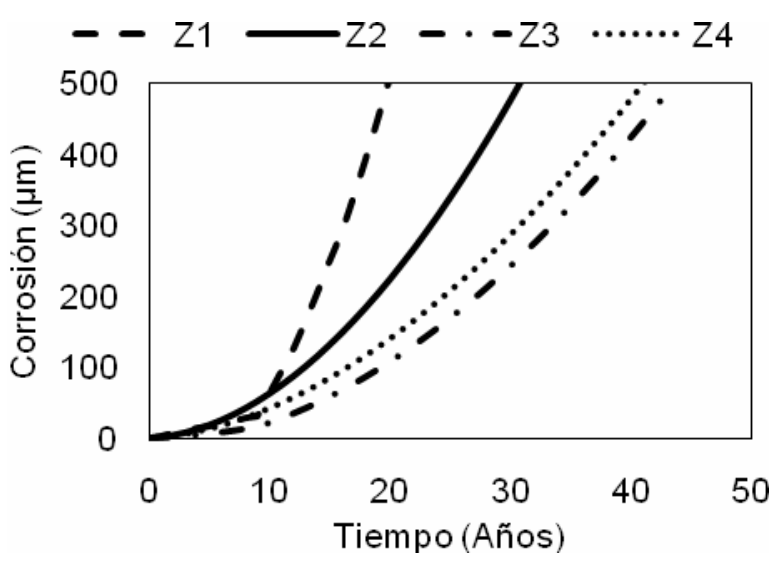

Fig. 2: Corrosión ( $\mu \mathrm{m})$ del zinc por zona.

En la Fig. 3 se describe el comportamiento de la vida útil por zonas. Para el acero galvanizado se estableció el comportamiento probabilístico de la vida útil de la estructura por zona considerando una distribución Weibull con un factor de forma dos (2) y el factor de vida característica de acuerdo a las funciones de degradación.

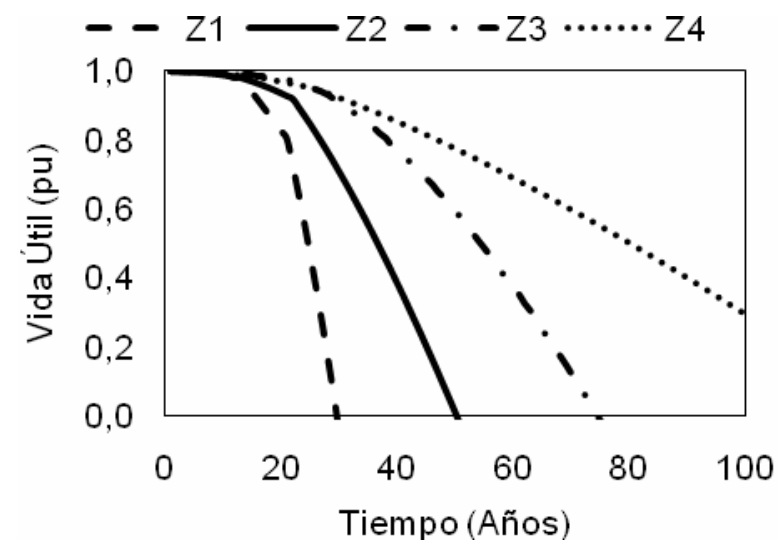

Fig. 3: Vida útil estructuras $3 / 16$ de pulgada.

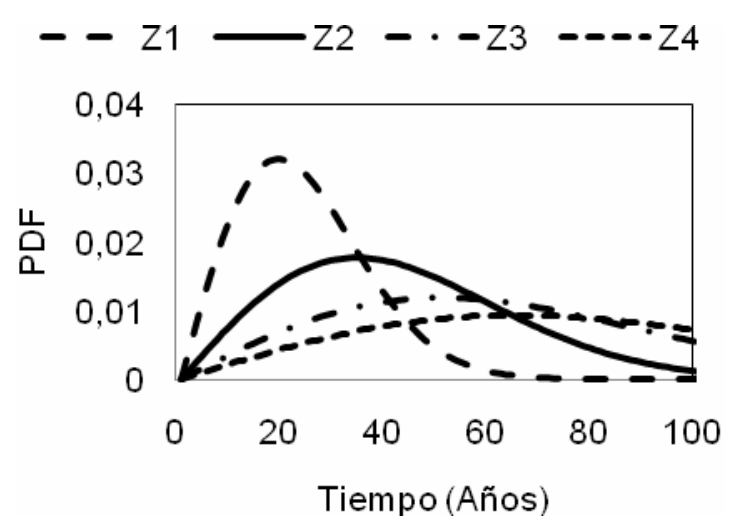

Fig. 4: Función distribución de probabilidad para la vida útil del acero galvanizado de $3 / 16$ de pulgada.

En la Fig. 4 se observa la función distribución de probabilidad. La disponibilidad se establece con base en las cadenas de Markov definidas para cada zona. 
En la Fig. 5 se observa el comportamiento de la disponibilidad considerando una capa máxima de 85 y 140 micras de zinc en estructuras de acero de 3/16.

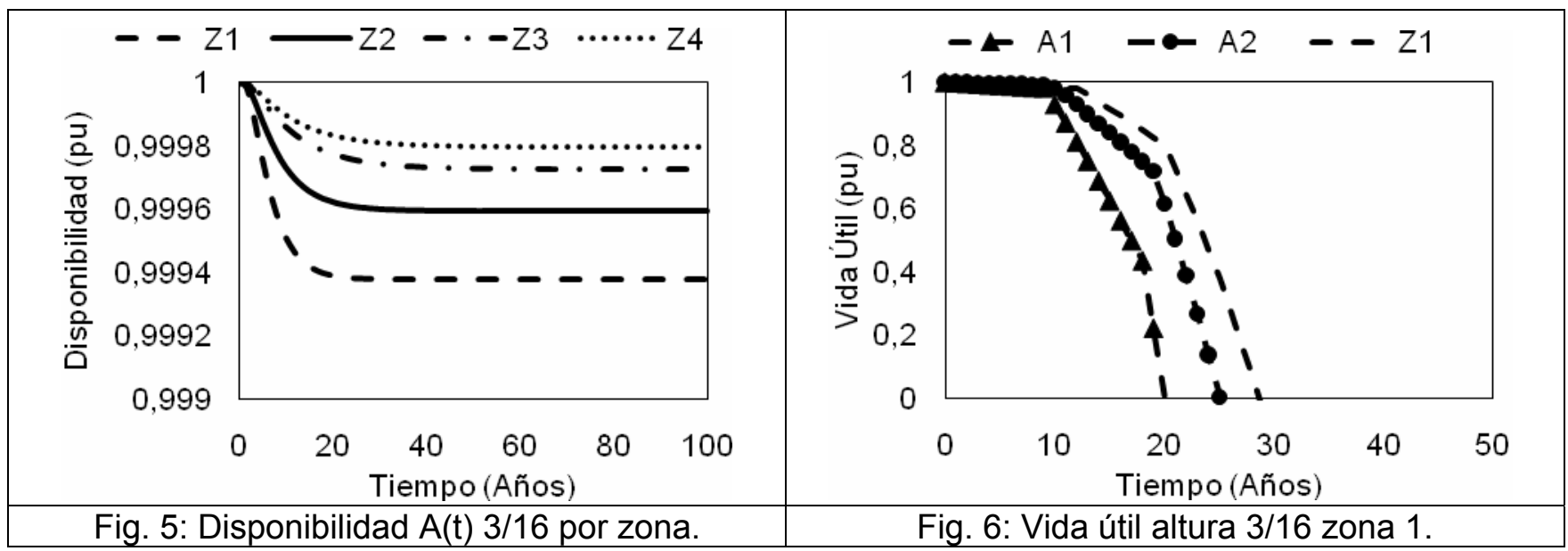

A su vez, se realizó una comparación de vida útil de las estructuras por nivel de altura para la zona 1, considerando dos tipos de escenarios. El primero corresponde a una zona con alta presencia de vientos y contaminación industrial moderada a alta (A1), el segundo sólo considerando un componente industrial moderado a alto (A2) comparado con la Zona (Z1). En la Fig. 6 se observa la incidencia de la altura sobre la vida útil de estructuras de $3 / 16$.

Por último se hallaron los valores óptimos de inspección, mantenimiento correctivo y mantenimiento preventivo. La simulación se realizó para cada una de las zonas y considerando las estructuras de $3 / 16$ con capas de 85 y 140 micras de zinc. En la Tabla 3 se observan los tiempos medios entre inspecciones (MTBI), mantenimientos correctos (MTBCM) y mantenimientos preventivos (MTBPM) de estructuras de $3 / 16$ pulgada.

Tabla 3: Tiempo medio entre inspecciones y mantenimientos 3/16.

\begin{tabular}{|l|c|c|c|c|}
\hline & Z1 & Z2 & Z3 & Z4 \\
\hline MTBCM & 11,93 & 24,35 & 47,64 & 89,58 \\
\hline MTBPM & 9,08 & 11,70 & 15,27 & 19,95 \\
\hline MTBI & 1,1435 & 1,1428 & 1,1425 & 1,1422 \\
\hline
\end{tabular}

Considerando los valores de vida útil del acero galvanizado y el comportamiento probabilístico se determina que la zona 1 , la más agresiva presenta una vida media cercana a la vida útil regulatoria reconocida en Colombia de 25 años. Sin embargo con los cambios regulatorios de vida útil la zona 1 sería la más afectada dado que se sugiere una vida útil regulatoria de 40 años lo que supondría que el valor adicional reconocido por contaminación atmosférica compense las actividades para cumplir la vida regulatoria. Incluso se observa como en zonas industriales impactaría el aumento de vida útil regulatoria. Caso contrario ocurre en las zonas 3 y 4 , los cuales presentan vidas superiores a los 50 años.

Los datos obtenidos de disponibilidad corresponden a estructuras de acero galvanizado para grosores de 85 y 140 micras de zinc. Se observa que la disponibilidad mínima es para la zona 1 . Sin embargo dichos valores no superan el límite de disponibilidad mínima exigida por la CREG (www.creg.gov.co) de 0,9977 para el caso de líneas de transmisión. El valor exigido por la CREG es referencial dado que supone para cualquier evento reconocido por la regulación y no sólo por contaminación atmosférica. Lo que sugiere implementar actividades preventivas y predictivas que no impliquen la indisponibilidad del activo.

Se observa que la frecuencia de inspección de todos los casos simulados es cercana a las 10,000 horas (14 meses), con variaciones que oscilan en 15 horas. Dicha frecuencia nos permite definir un 
plan de inspección anual, considerando que para el primer año se busca detectar imperfecciones de diseño y montaje y para las siguientes establecer posibles cambios en la agresividad de la atmósfera. Se establece que en la medida que se conozca el proceso de degradación la actividad principal a desarrollar es un mantenimiento correctivo basado en la condición de la estructura lo que disminuiría actividades preventivas y los costos asociados a estas, incluso con la implementación de grosores adicionales por medio de recubrimientos (pinturas, grasas, etc...) que incrementen la capa de protección y aumente el tiempo de intervención de la estructura considerando que en el general presentan actividades logísticas importantes.

Se estableció que la vida útil se ve fuertemente impactada por la altura y más en zonas altamente agresivas, lo que aumenta las frecuencias de mantenimiento y sus costos asociados. Es indispensable contar con protecciones adicionales, por el ejemplo la combinación de extragalvanizado y recubrimientos. La aplicación del recubrimiento se considera desde el inicio del montaje, debido a que existen mejor condiciones para su preparación e implementación. De lo contrario, si se implementará un tiempo después del montaje, no existirán las condiciones apropiadas y se incurriría en nuevas y frecuentes actividades de mantenimiento. Al considerar la vida útil regulatoria de referencia se incrementa el número de reposiciones que conlleven a cumplirla. En la práctica, para aquellas zonas en la cual la vida útil este muy cerca a la vida regulatoria, podría resolverse con buenas prácticas en el montaje y una cuidadosa inspección para identificar daños menores.

\section{CONCLUSIONES}

El trabajo realizado muestra la importancia de adelantar estudios en el área de la corrosión atmosférica, lo cual permite conocer los mecanismos de degradación de los materiales utilizados en el sector eléctrico en Colombia. La técnica de cadenas de Markov mostró ser una herramienta ágil para la simulación de las diferentes zonas y los respectivos escenarios de grosores, para evaluar la disponibilidad, los tiempos de inspección y mantenimiento, lo que permite tener herramientas para toma de decisiones sobre la pertinencia de implementar una estrategia en particular. Estableciendo la tasa de corrosión para los diferentes puntos de medida, es posible identificar y definir límites admisibles o de pérdida de función. De la misma manera identificar que tipos de mantenimientos a realizar, ya sea un galvanizado o un recubrimiento y su impacto dependiendo del grosor utilizado.

\section{AGRADECIMIENTOS}

Al Grupo de Corrosión y Protección, al Grupo de Manejo Eficiente de la Energía -GIMELpertenecientes a la Universidad de Antioquia, COLCIENCIAS, la Comisión de Regulación de Energía y Gas -CREG- y a las empresas donde fueron instaladas las muestras.

\section{REFERENCIAS}

Amari S. y L. McLaughlin; Optimal Desing of a Condition-Based Maintenance Model, Reliability and Maintainability, Annual Symposium - RAMS IEEE, 528- 533, Los Angeles, California - USA, Ene 2629 (2004).

Anders G.J., J. Endrenyi y C. Yung; Risk-based planner for asset management, IEEE Computer Applications in Power: 14 (4), 20-26 (2001).

CIC, Concepto técnico de la contaminación salina en conductores ACSR, aisladores y postes de concreto en transmisión y distribución de energía, Bogotá, Colombia (2004).

COLCIENCIAS/CREG, "Impacto de la corrosividad atmosférica sobre la infraestructura del SEC y sobre los costos AOM", Proyecto de Investigación, Universidad de Antioquia, Grupo de Corrosión y Protección - Grupo de Manejo Eficiente de la Energía -GIMEL, Junio (2008).

Endrenyi J. y G.J. Anders; Aging, maintenance, and reliability, IEEE power \& energy magazine: 4(3), 59-67 (2006). 
Grall A., L. Dieulle, C. Bérenguer y M. Roussignol; Continuous-Time Predictive-Maintenance Scheduling for a Deteriorating System, IEEE Transactions on Reliability: 51(2), 141-150 (2002).

Gustavsen B. y L. Rolfseng; Asset management of wood pole utility structures, Electrical Power and Energy Systems, doi:10.1016/j.ijepes.2005.08.005 (en línea), 27 (9-10), 641-646 (2005) (fecha de consulta: Febrero 2007), acceso libre, http://www.sciencedirect.com/science/journal/01420615.

IEC 61165; Application of Markov techniques, Second edition, 1-76, Geneva, Switzerland (2006).

ISO 9223; Corrosion of Metals and Alloys -Corrosivity of atmospheres - Classification, 1-18, Geneva, Switzerland (1992).

Jiang, Y., J. McCalley y T. Van Voorhis; Risk-Based Resource Optimization for Transmission System Maintenance; IEEE Transactions on Power Systems: 21(3), 1191-1200 (2006).

Kutz M.; "Handbook of Environmental Degradation of Materials", 1-581, William Andrew Publishing, Norwich NY - USA (2005).

Lefebvre Y.; Using Equivalent Failure Rates to Assess the Unavailability of an Ageing System, Proceedings Annual Reliability and Maintainability Symposium IEEE, 82-88, Tampa, Florida - USA, Ene 27-30 (2003).

Linares L., N. Taborda, L. Zambrano y O. Pérez; Failures Analysis by Corrosion in Power Conductors of Aluminum Alloys in Coastal-Lacustrian Environments, Transmission \& Distribution Conference and Exposition: Latin America, 2006. TDC '06. IEEE/PES, 1-6, Caracas, Venezuela, Ago 15-18 (2006).

Mango, J.; Coating Radiators for Corrosion Resistance: Galvanized vs. Painted - User's Perspective, Transmission and Distribution Conference and Exposition, 2001 IEEE/PES, 1118-1121, Atlanta, USA, Oct 28 - Nov 2 (2001).

Maya, J.; Metodología para el manejo integral de la corrosión en Líneas de transmisión de energía eléctrica; III Congreso CIER de la Energía CONCIER, Abastecimiento Energético Regional -Retos y Perspectivas-, Medellín Colombia, Nov 27-30 (2007).

Minotas J.C., C.E. Arroyave, A. Valencia y R. Pérez; Avances en los estudios de corrosión atmosférica en Colombia, Revista Facultad de Ingeniería Univ. de Antioquia: 8 (3), 32-42 (1996).

Molina J.D.; "Impacto de la corrosión atmosférica sobre la infraestructura de Líneas de Transmisión en Colombia", Tesis de Maestría, Facultad de Ingeniería, Univ. de Antioquia, Medellín, Colombia (2008).

Siqueira, I.P.; Optimum Reliability-Centered Maintenance Task Frequencies for System Equipments, 8th International Conference on Probabilistic Methods Applied to Power Systems PMAPS IEEE, 162167, Ames lowa - USA, Sep 12 -16 (2004).

Tapias, J.; "Modelo para o gerenciamento de ativos de transmissão de energia elétrica", Tesis de Maestría, Pós-Graduação em Engenharia Elétrica, Univ. Federal de Santa Catarina, Florianópolis, Brazil (2004).

UPME-Unidad de Planeación Minero Energética; "Plan de Expansión de Referencia Generación Transmisión 2008-2022”, 1-203, Ministerio de Minas y Energía, Bogotá, Colombia (2008).

Veleva L. y R.D. Kane; "Atmospheric Corrosion, Corrosion: Fundamentals, Testing, and Protection", Vol 13A, 1-2597, ASM Handbook, ASM International, USA (2003).

Vera R., D. Delgado y B. Rosales; Effect of atmospheric pollutants on the corrosion of high power electrical conductors: Part 1. Aluminium and AA6201 alloy, doi:10.1016/j.corsci.2005.11.012, (en 
línea), 48, 2882-2900 (2006), (fecha de consulta: Febrero 2007), acceso libre, http://www.sciencedirect.com.

Vera R., D. Delgado y B. Rosales; Efect of atmospheric pollutants on the corrosion of high power electrical conductors - Part 2. Pure copper, doi:10.1016/j.corsci.2006.10.031 (en línea), 49, 23292350, 2007, (fecha de consulta: Febrero 2007), acceso libre, http://www.sciencedirect.com.

Vera, R.M. y F.J. Cañas; Comportamiento frente a la corrosión en ambiente marino de acero galvanizado y acero galvanizado pintado (Duplex). Inf. Tecnol.: 16(4), 53-58. ISSN 0718-0764, (2005). 